\title{
Study on the Influence of Vehicle Sound Source Height on Traffic Noise Forecast
}

\author{
Xintan $\mathrm{Ma}^{\mathrm{a}}$, Guopeng Chang ${ }^{\mathrm{b}}$ \\ School of Vehicle and Transportation Engineering, Henan University of Science and Technology, \\ Henan Luoyang 471003, China, \\ a maxintan@163.com, b2468518077@qq.com
}

Keywords: Noise model; Sound source height; Integrated noise; Sound pressure level.

\begin{abstract}
At present ,the prediction result of highway traffic noise is quite different from the actual situation, the main reason of which is that the prediction model of the sound source is not perfect. This paper considers the actual height of highway sound source, uses acoustic simulation software to build a sound source model whose vertical height is consistent with the actual situation ,analysis the prediction results of sound receiving points and discovers that: compared with the equivalent simple sound source model, that having considered the sound source height much more accord with the actual highway situation so that the prediction accuracy will be improved, which will provide reference to the road traffic noise control.
\end{abstract}

\section{Introduction}

The increasing number of car ownership makes the problem of traffic noise pollution deeply disturbing to our life, so it is urgent to prevent the road noise pollution. Highway traffic noise model is the basis of road noise research, literature [1] the FHWA, RLS90 specification model predicted results are analyzed, there are too many heavy vehicles, the standard model of noise prediction error more bigger. Literature [2-3] takes the urban first road as the research object, and finds that the absolute error of the guidance model is $3.3-3.6 \mathrm{~dB}$, and the absolute error of night is $-3.4-0.29 \mathrm{db}$.The current traffic noise model, with a line close to the ground along the equivalent traffic line source model to simulate road traffic source (also called equivalent simple sound source model [4]), the equivalent sound source model ignores the actual source height on road noise source definition model and the influence of sound pressure level prediction, The equivalent sound source model neglects the influence of the actual height of sound source on the definition of sound source and the prediction of sound pressure noise model, which make the sound the sound pressure have a greater difference between predicted values and the actual situation.

Based on the equivalent sound source model, this paper considers the actual height of sound source under three kinds of road conditions: heavy vehicle, light vehicle and light, medium and heavy vehicle. Based on the traffic noise model which is consistent with the sound source, the integrated noise and sound pressure level of three kinds of road conditions are calculated and compared with the prediction results of equivalent sound source under the same conditions.

\section{Highway Traffic Noise Source}

Only when the sound source model is close to the actual situation of the road can the sound pressure level prediction at the sound point be more accurate. Processing methods for noise source model both at home and abroad, the highway traffic vehicle type is divided into light, medium and heavy vehicles, and the height of the sound source were $0.35 \mathrm{~m}, 0.85 \mathrm{~m}, 1.5 \mathrm{~m}$, in order to calculate the traffic noise generally take the average height $1 \mathrm{~m} \mathrm{[5].The} \mathrm{location} \mathrm{of} \mathrm{the} \mathrm{sound} \mathrm{source} \mathrm{is} \mathrm{determined} \mathrm{by} \mathrm{means}$ of the equivalent driving line, located near the center line of the road. If the traffic flow is greater than 1000 vehicles/hour, it can be regarded as a line sound source [6].According to road noise measurement method from the point of reference for the equivalent traffic line $r_{0}$ sound pressure level spectrum, then according to the standard of environmental noise sound pressure level and sound 
power level, the relationship between point get by the sound of sound pressure level and sound power [7] are given as follows:

$$
\begin{gathered}
L_{p}=L_{w}+\sum \Delta_{\text {propagation factors }} \\
L_{w}=L_{r_{0}}+10 \lg _{r_{0}}+3
\end{gathered}
$$

$\mathrm{L}_{\mathrm{r}_{0}}$ - the sound pressure level at the reference point; $\mathrm{L}_{\mathrm{W}}$ - sound source sound power level; $\sum \Delta_{\text {propagation factors }}$ the sum of correction and attenuation of noise propagation process; $r_{0^{-}}$ the distance between the sound source and the reference point; $\mathrm{L}_{\mathrm{p}}$ - sound pressure level.

The combined formula (1) and (2) can obtain the sound pressure level:

$$
\begin{gathered}
\mathrm{L}_{\mathrm{p}}=\mathrm{L}_{\mathrm{r}_{0}}+10 \lg _{\mathrm{r}_{0}}+\sum \Delta_{\text {propagation factors }}+3 ; \\
\sum \Delta_{\text {propagation }}=\Delta_{\text {geo }}+\Delta_{\text {air }}+\Delta_{\text {ground }}+\Delta_{\text {barrier }}+\Delta_{\text {refection }} ;
\end{gathered}
$$

$\Delta_{\text {geo }}$ - diffusion attenuation; $\Delta_{\text {air }}$ - air absorption attenuation; $\Delta_{\text {ground }}$ - ground absorption attenuation; $\Delta_{\text {barrier- }}$ - the attenuation of the noise barrier; $\Delta_{\text {refection }}$ - ground reflection correction.

If the measured distance of the source $r_{0}$ meters is the reference point, the sound pressure level of the sound point can also be obtained by formula (5).

$$
L_{p}=L_{r_{0}}-10 \lg \left(\frac{r}{r_{0}}\right) \text {; }
$$

$L_{r_{0}}$-refer to the sound pressure level; $10 \lg \left(\frac{r}{r_{0}}\right)$ - source spread geometry attenuation infinite long term.

\section{The Sound Source Model}

Traffic sound source location and accurate definition is very important, in the study of the past all the traffic noise close to the ground of the equivalent driveway, ignores the actual height of the source, this is a greater difference between predicted results and the actual situation of one of the important reasons.

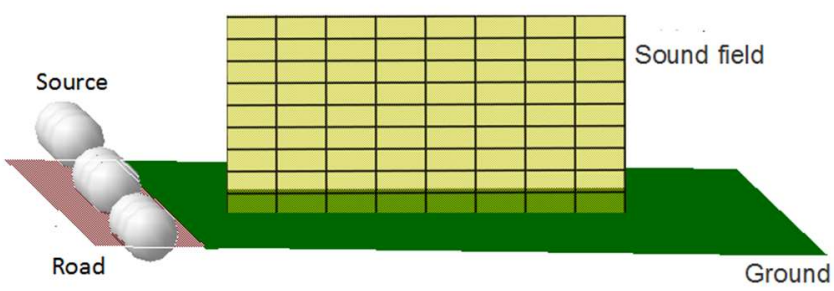

Fig. 1 Traffic noise model without a sound barrier

\subsection{Modeling Parameters and Design Scheme.}

A road model with a length of $14.5 \mathrm{~m}$ was established. The distance between vehicles was $30 \mathrm{~m}$ and the road length was $300 \mathrm{~m}[8]$.According to the three kinds of road conditions, three calculation schemes: scheme 1, when all the traffic conditions are heavy, 9 points are selected to define the sound source, and the sound source height is $1.5 \mathrm{~m}$; scheme 2, when all the light vehicle road conditions, 9 points are selected to define the sound source, and the sound source height is $0.35 \mathrm{~m}$; Scheme 3 : when the combination of light, medium and heavy vehicles is mixed, the sound source height is $0.35 \mathrm{~m}$, $0.85 \mathrm{~m}$ and $1.5 \mathrm{~m}$ respectively. When all three models exist, it is referred to as the three sound source model, as shown in FIG.1, with a distance of $20 \mathrm{~m}$ from shoulder and height of $2 \mathrm{~m}$.According to the relevant standards [9], the frequency response curve of the reference point of the equivalent driving line $r_{0}=7.5 \mathrm{~m}$ is shown in FIG.2. 


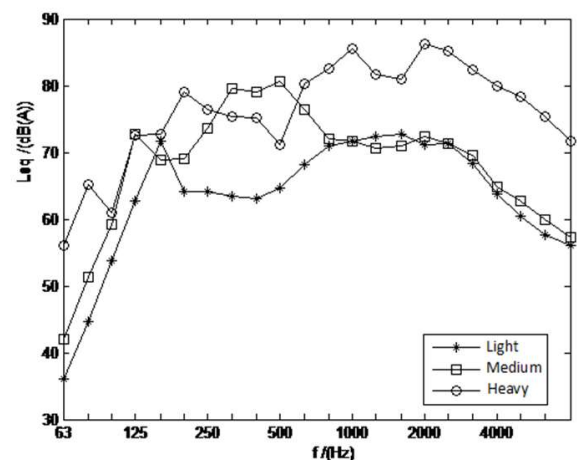

Fig. 2 the sound pressure level spectrum of different vehicle models

Having obtained the sound pressure level value of the reference point, using the formula(5) to obtain the sound pressure level spectrum of the three vehicle acoustic source locations through the sound pressure level spectrum of the reference point, and using the formula(2) to obtain the sound power level spectrum of the light, medium and heavy vehicle acoustic model, as shown in table 1, and defining parameters such as air temperature, spectrum absorption characteristics of the ground, In Virtual. lab, the frequency spectrum of sound sources of light, medium and heavy vehicles at the site of sound is calculated.

Table 1. the sound power level of different vehicle sound sources

\begin{tabular}{|c|c|c|c|}
\hline $\begin{array}{c}1 / 3 \\
\text { Octave }(\mathrm{Hz})\end{array}$ & $\begin{array}{l}\text { Light Vehicle } \\
\text { Sound Power Level dB(A) }\end{array}$ & $\begin{array}{l}\text { Medium vehicle } \\
\text { Sound Power Level dB(A) }\end{array}$ & $\begin{array}{c}\text { Heavy Vehicle } \\
\text { Sound Power Level } d B(A)\end{array}$ \\
\hline 63 & 47.75 & 53.75 & 67.55 \\
\hline 80 & 56.33 & 63.27 & 76.75 \\
\hline 100 & 65.50 & 70.92 & 72.58 \\
\hline 125 & 74.25 & 84.25 & 84.25 \\
\hline 250 & 75.92 & 85.25 & 87.98 \\
\hline 315 & 75.08 & 91.17 & 86.92 \\
\hline 400 & 74.67 & 90.75 & 86.75 \\
\hline 500 & 76.08 & 92.17 & 82.75 \\
\hline 800 & 82.58 & 83.58 & 94.25 \\
\hline 1000 & 83.42 & 83.42 & 96.98 \\
\hline 1600 & 84.25 & 82.58 & 92.53 \\
\hline 2000 & 82.67 & 83.38 & 97.83 \\
\hline 2500 & 83.0 & 83.0 & 96.75 \\
\hline 4000 & 75.50 & 76.58 & 91.67 \\
\hline
\end{tabular}


The acoustic pressure level spectrum at the same sound $\operatorname{point}(\mathrm{R}=20 \mathrm{M})$ is calculated by using the sound pressure level superposition formula of three kinds of vehicles. Using a formula(2) to obtain a single acoustic power level spectrum as shown in Table 2.

Table 2. the equivalent acoustic power level of a single source model

\begin{tabular}{cccccc}
\hline $\begin{array}{c}1 / 3 \text { Octave } \\
(\mathrm{Hz})\end{array}$ & $\begin{array}{c}\text { Sound Power Level } \\
\mathrm{dB}(\mathrm{A})\end{array}$ & $\begin{array}{c}1 / 3 \text { Octave } \\
(\mathrm{Hz})\end{array}$ & $\begin{array}{c}\text { Sound Power Level } \\
\mathrm{dB}(\mathrm{A})\end{array}$ & $\begin{array}{c}1 / 3 \text { Octave } \\
(\mathrm{Hz})\end{array}$ & $\begin{array}{c}\text { Sound Power Level } \\
\mathrm{dB}(\mathrm{A})\end{array}$ \\
\hline 63 & 67.78 & 315 & 92.46 & 1250 & 94.20 \\
80 & 76.99 & 400 & 92.29 & 1600 & 93.55 \\
100 & 75.33 & 500 & 92.74 & 2000 & 98.14 \\
125 & 87.48 & 630 & 93.49 & 2500 & 97.11 \\
160 & 87.74 & 800 & 94.88 & 3150 & 94.46 \\
200 & 91.09 & 1000 & 97.83 & 4000 & 91.91 \\
250 & 90.03 & & & & \\
\hline
\end{tabular}

\section{Analysis of Simulation Results.}

In the absence of sound barrier, when the road traffic condition is all heavy vehicles, the sound pressure level frequency response curve of the sound point is shown in figure 3.

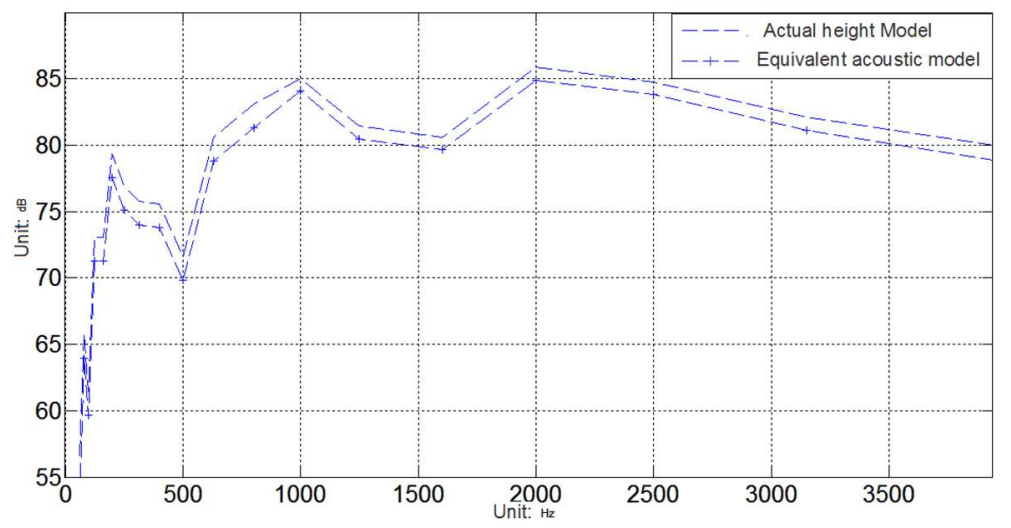

Fig. 3 The sound pressure level frequency response curve of heavy traffic condition without sound barrier

Consider the height of the sound source model and the noise equivalent sound source model integrated sound in the point of $93.27 \mathrm{~dB}$ and $92.04 \mathrm{~dB}$, respectively, the comprehensive noise difference of $1.23 \mathrm{~dB}$, its point of the sound pressure level (63-4000), frequency domain within the scope of sound pressure level difference value of $0.97 \mathrm{~dB} 1.77 \mathrm{~dB}$, at $80 \mathrm{~Hz}-315 \mathrm{~Hz}$ difference is bigger, about $1.77 \mathrm{~dB}$; The difference between the difference value of $1000 \mathrm{~Hz}$ and the value of $1000 \mathrm{~Hz}$ is about $1 \mathrm{~dB}$, and the spectrum difference is shown in FIG. 4.

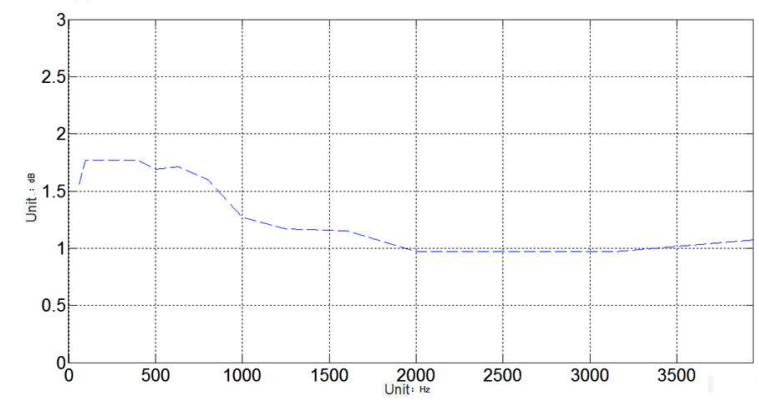

Fig.4 The spectral difference of the acoustic pressure level of two acoustic source models without sound barrier 
Using the same method to calculate when full for light vehicle traffic, consider the height of the sound source model and the equivalent sound source model integrated sound in the point of noise were $77.62 \mathrm{~dB}$ and $77.62 \mathrm{~dB}$, integrated the difference value of $0.86 \mathrm{~dB}$ of noise, the noise points of different frequency sound pressure level difference value in $0.6 \mathrm{~dB}-1.5 \mathrm{~dB}$;At $80 \mathrm{~Hz}$, the maximum difference is $1.89 \mathrm{~dB}$. When light, medium and heavy-duty trucks are road conditions, consider the height of the sound source three comprehensive noise source model and the equivalent sound source model were $89.12 \mathrm{~dB}$ and $89.12 \mathrm{~dB}$, integrated the difference value of $0.35 \mathrm{~dB}$ of noise, the noise points of different frequency sound pressure differential value in $0.05 \mathrm{~dB}-0.42 \mathrm{~dB}$.

Comparing the prediction results of sound pressure level and integrated noise of three schemes, it is found that the model considering the sound source height is less different from the equivalent sound source model. When the road is all light or heavy vehicles, considering the source height model and the equivalent source prediction results are more different, especially when there are more heavy vehicles or all heavy vehicles.

\section{Conclusion}

1. The sound source height is an important factor that influences the accuracy of the sound source model, which directly influences the prediction results of the sound pressure level spectrum and comprehensive noise.It is more accurate to establish a sound source model which is consistent with the sound source.

2, light, medium and heavy vehicle driving road conditions, consider the height of the sound source model and the equivalent sound source model predicted results difference is small, the forecast results were different when all were light or heavy. The equivalent single-source model is reasonable under certain conditions, but its scope is limited.

3. In the case of silent barrier, the difference between the model with the actual height of the sound source and the equivalent single source model is $0.35 \mathrm{~dB}-1.23 \mathrm{~dB}$.

\section{References}

[1]. Cao Li-na,Shang Yong,Lu Lin-guo.Comparison of FHWA, RLS90 and MOT Model for Highway Traffic Noise Prediction[J]. Journal of Zhengzhou University(Engineering Science), 2014,35(3):106-110.

[2]. Fan Dong-ping,Zhang Yu-huan, et al.Comparison among results of road traffic noise prediction models[J].Chinese Journal of Environmental Engineering, 2013.6:2392-2395.

[3]. Fan Dong-ping.Road traffic noise prediction model analysis and empirical research[D].Guangzhou:Guangdong University of Technology,2012:14-15.

[4]. Ma Xin-tan,Feng Shi-chang.Simulation and Analysis on the Traffic Noise Models of Four-Lane Highway[J].Machinery Design \& Manufacture, 2017,(z1):106-109.

[5]. Zhang Yu-fen,Qian Bing-hua,Dai Ming-xin.Transportation and Environmental Protection[M].Beijing:China Communications Press,2003:106-108.

[6]. Zhao Chun-lai,Ma Xin-tan,Guo Zhi-jun. Optimization Design of Highway Sound Barrier Based on the Effective Length of Sound Source[J].Noise and Vibration Control,2011(1):153-156.

[7]. Zhao Chun-lai,Ma Xin-tan,Guo Zhi-jun.Parametric Analysis and Optimization Design of Road Noise Barrier[J].Journal of Henan University of Science \& Technology(Natural Science) ,2010,31(4):23-27.

[8]. Li Lei.Study on Doppler Effect of Vehicle Noise and the Optimization Method of Noise Barrier[D]. Henan University of Science and Technology,2015:30-31.

[9]. GB3096-2008, Environmental quality standard for noise[S].4-6. 